\title{
Effects of infrasound on the growth of bone marrow mesenchymal stem cells: A pilot study
}

\author{
RENHONG HE and JIANZHONG FAN \\ Department of Rehabilitation Medicine, Nanfang Hospital, Southern Medical University, \\ Guangzhou, Guangdong 510515, P.R. China
}

Received November 2, 2013; Accepted May 9, 2014

DOI: $10.3892 / \mathrm{mmr} .2014 .2508$

\begin{abstract}
Poor viability of transplanted bone marrow mesenchymal stem cells (BMSCs) is well-known, but developing methods for enhancing the viability of BMSCs requires further investigation. The aim of the present study was to elucidate the effects of infrasound on the proliferation and apoptosis of BMSCs, and to determine the association between survivin expression levels and infrasound on BMSCs. Primary BMSCs were derived from Sprague Dawley rats. The BMSCs, used at passage three, were divided into groups that received infrasound for 10, 30, 60, 90 or $120 \mathrm{~min}$, and control groups, which were exposed to the air for the same durations. Infrasound was found to promote proliferation and inhibit apoptosis in BMSCs. The results indicated that 60 min was the most suitable duration for applied infrasound treatment to BMSCs. The protein and mRNA expression levels of survivin in BMSCs from the two treatment groups that received $60 \mathrm{~min}$ infrasound or air, were examined by immunofluorescence and quantitative polymerase chain reaction. Significant differences in survivin expression levels were identified between the two groups, as infrasound enhanced the expression levels of survivin. In conclusion, infrasound promoted proliferation and inhibited apoptosis in BMSCs, and one mechanisms responsible for the protective effects may be the increased expression levels of survivin.
\end{abstract}

\section{Introduction}

Stem cells possess the potential to differentiate into specific cell phenotypes, and numerous recent studies have focused on stem cell properties. Bone marrow mesenchymal stem cells (BMSCs) are of interest as these cells can be easily isolated from a small aspirate of bone marrow and have few ethical

Correspondence to: Mr. Jianzhong Fan, Department of Rehabilitation Medicine, Nanfang Hospital, Southern Medical University, 1838 Guangzhou North Road, Guangzhou, Guangdong 510515 , P.R. China

E-mail: hereon@163.com

Key words: infrasound, bone marrow mesenchymal stem cells, survivin limitations (1). Therefore, BMSCs are considered the most suitable stem cells for research (1). A number of studies have induced BMSC differentiation into different cell types in vitro, including chondrocytes, osteoblasts, adipocytes and myoblasts (2). Another area of research concerns the replacement of impaired tissue through transplantation of BMSCs $(3,4)$. Studies have indicated that BMSCs significantly enhance motor function recovery when transplanted into animal models with cerebral infarction, subcortical capsular infarction and traumatic brain injury $(3,4)$. BMSCs have shown great promise in tissue repair; however, the poor viability of transplanted BMSCs has limited the therapeutic potential $(5,6)$. Therefore, enhancing the survival of BMSCs requires further investigation. Certain studies have found that continuous perfusion stimulates the proliferation of human BMSCs. Furthermore, reducing the fluid flow rate while maintaining a constant peak fluid shear stress is associated with alterations in BMSC proliferation $(7,8)$. These studies suggest that proliferation of BMSCs is affected by mechanical stimulation.

Infrasound refers to inaudible noise at a frequency $<20 \mathrm{~Hz}$ and may be considered to consist of mechanical vibrations that are difficult to detect with the human ear (9). The effects of infrasound are disputed, with some researchers suggesting that infrasound is hazardous to the human body $(10,11)$. Infrasound frequencies at $8 \mathrm{~Hz}, 130 \mathrm{~dB}$, have been demonstrated to impair the rat myocardium and induce elevated levels of intracellular calcium ions in the hippocampal cells of the rat brain $(10,11)$. However, a previous study has shown that infrasound with the sound pressure level $<90 \mathrm{~dB}$ improved the motor function of rats following middle cerebral artery occlusion (12). Thus infrasound with a high sound pressure level $>90 \mathrm{~dB}$ may impair tissue, while low pressure levels $<90 \mathrm{~dB}$ may be beneficial. The aim of the present study was to investigate whether infrasound with a low sound pressure level $<90 \mathrm{~dB}$ alters the proliferation and apoptosis of BMSCs.

Survivin is a member of the inhibitor of apoptosis (IAP) family and is specifically expressed at the G2/M phase of the cell cycle. Histological examination has revealed that survivin is expressed in growing tissues, such as the thymus, testis and the intestine of adult mice, and numerous embryonic tissues, but not in mature tissues (13). IAP proteins comprise a highly conserved gene family that prevent cell death in response to a variety of stimuli (14). Survivin has been implicated in multiple 
essential functions, including cell division, programmed cell death or apoptosis, the cellular stress response and checkpoint mechanisms of genomic integrity (15). One study found $\sim 83 \%$ BMSCs expressed survivin, with more marked staining in the nucleus than in the cytoplasm (16).

In the present study, the effects of infrasound on the proliferation and apoptosis of BMSCs were analyzed. Evidence indicates that survivin may mediate changes in BMSC biological behavior following infrasound treatment (14-16). Thus the present study was designed to investigate the association of survivin expression levels with the effects of infrasound on BMSCs at the molecular level.

\section{Materials and methods}

Infrasound device. The infrasound device (Chi- $\left.8^{\mathrm{TM}}\right)$ used in the present study was manufactured by the Chi Institute (San Juan Capistrano, CA, USA). The device consisted of two sections containing the transmitting probe and the mainframe. The infrasound device consists of three options, for which option thre was used for this study, producing a frequency of 4-20 Hz and a sound pressure level of 79-86 dB. A previous study suggested that the this setting exerted a protective effect on rats with cerebral ischemia-reperfusion injury (12).

Animal experiments and ethical approval. BMSCs were harvested from female Sprague Dawley rats (weight, $100 \mathrm{~g}$; age, 4 weeks) provided by the Animal Center of the Southern Medical University (Guangzhou, China). All experimental procedures on the rats were approved by the Animal Ethics Committee of Nanfang Hospital (Permit number: NFYY20120128; Guangzhou, China). The rats were anesthetized with $10 \%$ chloral hydrate and then sacrificed by cervical dislocation.

BMSC harvest and culture. The rat femora and tibiae were aseptically excised and the epiphyses of the bones were removed. The bone marrow was flushed from the shaft with Dulbecco's modified Eagle's medium (DMEM)/F12 (Hyclone, Waltham, MA, USA) using a 20 -gauge needle. The bone marrow suspension was disaggregated by pipetting several times and the cells were collected by centrifugation $(2,200 \mathrm{x} \mathrm{g}$, $5 \mathrm{~min}$ (17). The cells were then cultured in DMEM/F12 with $10 \%$ fetal bovine serum (Gibco-BRL, Carlsbad, CA, USA) containing $100 \mu \mathrm{l} / \mathrm{ml}$ penicillin-streptomycin in $25 \mathrm{~cm}^{2}$ cell culture flasks (Corning Inc., Acton, MA, USA). The cells were incubated at $37^{\circ} \mathrm{C}$ in a $5 \% \mathrm{CO}_{2}$ atmosphere. After $48 \mathrm{~h}$, non adherent cells were removed using phosphate buffer saline (PBS; Boster Biological Tech Ltd, Wuhan, China) to rinse the cells. The culture mediun was replaced every second or third day. When the cells reached $85 \%$ confluence, the primary culture was subcultured 1:2; third-passage BMSCs were used in the present study.

Characterization of BMSCs by immunophenotype. The third-passage BMSCs were dissociated with $0.25 \%$ trypsin and suspended at a concentration of $5 \times 10^{5}$ cells $/ \mathrm{ml}$ in PBS. A volume of $1 \mathrm{ml}$ cell suspension was pipetted into a $1.5 \mathrm{ml}$ tube. CD29-PE/CY5, CD45-fluorescein isothiocyanate (FITC) and CD90-APC/CY7 (BioLegend, Inc., San Diego, CA,
USA) were added to the respective tubes and the tubes were incubated for $30 \mathrm{~min}$ at room temperature in the dark. The control group was absent of these antibodies. Flow cytometric analysis was performed to measure the positive rate using a BD LSRFortessa and Facsdiva 6.2 software (BD Biosciences, San Jose, CA, USA).

Infrasound and control treatments. The third-passage BMSCs were detached with $0.25 \%$ trypsin and resuspended in a $5 \mathrm{ml}$ tube with DMEM/F12 in 10\% fetal bovine serum, then divided into infrasound and control groups. The infrasound groups were exposed to infrasound and the control groups were exposed to air. The durations of the two treatments were 10 , 30, 60, 90 and $120 \mathrm{~min}$. Following the interventions, the cells were immediately incubated at $37^{\circ} \mathrm{C}$ in a $\mathrm{CO}_{2}$ atmosphere for $72 \mathrm{~h}$. The BMSCs in each group were seeded in 96-well plates for the proliferation assay or in a $6 \mathrm{~cm}$ culture dish for the apoptosis assay. In addition, BMSCs from the two groups that received $60 \mathrm{~min}$ infrasound or air treatment were seeded in 24-well plates containing 1x1 mm coverslips for immunofluorescence analysis and 6-well plates for quantitative polymerase chain reaction (qPCR).

Evaluation of cell proliferation by cell counting kit (CCK) assay. In order to evaluate the proliferation ability of the BMSCs, a CCK (Dojindo, Kunamoto, Japan) assay was performed. A volume of $10 \mu \mathrm{l} \mathrm{CCK}$ reagent was added to the wells and the cells were then incubated at $37^{\circ} \mathrm{C}$ for $3 \mathrm{~h}$ until the emergence of an orange supernatant. The optical density (OD) was measured at $630 \mathrm{~nm}$.

Measurement of apoptotic cells. The BMSCs were dissociated with $0.25 \%$ trypsin, centrifuged at 2,200 $\mathrm{x}$ g for $5 \mathrm{~min}$, then suspended at a concentration of $5 \times 10^{5}$ cells $/ \mathrm{ml}$ with PBS. The Annexin V-FITC Apoptosis Assay kit (Nanjing Keygen Biotech. Co., Ltd., Nanjing, China) was used for the assay. The cell suspension was rinsed with PBS and centrifuged again, and the cells were then suspended in $500 \mu \mathrm{l}$ buffer. Annexin V-FITC and propidium iodide were added to the cell suspension and incubated for $10 \mathrm{~min}$ at room temperature in the dark according to the manufacturer's instructions. Flow cytometry was conducted to analyze the results.

Survivin immunohistochemistry. Immunohistochemistry of survivin expression was performed on only the $60 \mathrm{~min}$ infrasound and control groups. The BMSCs on coverslips were fixed with fresh $4 \%$ paraformaldehyde for $30 \mathrm{~min}$. Subsequent to washing with PBS three times and permeabilization with 0.25\% TX-100 for $20 \mathrm{~min}$, the cells were blocked for $40 \mathrm{~min}$ at room temperature with $5 \%$ bovine serum albumin (Boster Biological Technology, Ltd., Wuhan, China). The cells were then incubated at $4^{\circ} \mathrm{C}$ overnight with primary antibody against survivin (Bioworld Technology, Inc., St. Louis Park, MN, USA). Following washing, the cells were incubated at room temperature with secondary Alexa Fluor 594 goat anti-rabbit antibodies (Invitrogen Life Technologies, Carlsbad, CA, USA) for $1 \mathrm{~h}$. The cells were washed again and incubated with DAPI (Boster Biological Technology, Ltd.) for $5 \mathrm{~min}$. The fluorescent signals were visualized under a confocal laser microscope (Olympus, Tokyo, Japan). 
Table I. Primers used for quantitative polymerase chain reaction.

\begin{tabular}{llrr}
\hline Gene & \multicolumn{1}{c}{ Primer sequence } & Length (bp) & Accession no. \\
\hline$\beta$-actin & Forward: 5'-TTGGTGGCTCTATCCTGGCCTC-3' & 131 & NM_031144.2 \\
Survivin & $\begin{array}{l}\text { Reverse: 5'-AAACGCAGCTCAGTAACAGTCCG-3' } \\
\text { Forward: 5'-ACTGCCCTACCGAGAATGAG-3' } \\
\text { Reverse: 5'-GAGTGCTTCCTATGCTCCTCT-3' }\end{array}$ & 109 & NM_022274.1 \\
\hline
\end{tabular}

bp, base pairs.

qPCR of survivin $m R N A$. The BMSCs were harvested and homogenized for RNA extraction with TRIzol ${ }^{\mathrm{TM}}$ reagent (Invitrogen Life Technologies). Messenger RNA was reverse-transcribed to cDNA using Primescript RT Reagent kit (Takara Bio, Inc., Shiga, Japan). qPCR was then conducted to measure the survivin mRNA expression level. The expression levels of the $\beta$-actin housekeeping gene were measured as a control. The primer sequences are shown in Table I. A volume of $2 \mu 1$ total cDNA from each sample was amplified in a final volume of $25 \mu \mathrm{l}$ reaction mixture containing $\mathrm{SYBR}^{\circledR}$ Green I (Takara Bio Inc., Shiga, Japan) using the Applied Biosystems 7500 Fast Real-Time PCR system (Applied Biosystems, Inc., Foster City, CA, USA). The cycling conditions were as follows: $95^{\circ} \mathrm{C}$ for $30 \mathrm{sec}, 40$ cycles at $95^{\circ} \mathrm{C}$ for $5 \mathrm{sec}$ and $34 \mathrm{sec}$ at $60^{\circ} \mathrm{C}$.

Statistical analysis. All the results are presented as the means \pm standard deviation for each group. The data were analyzed using SPSS software (SPSS, Inc., Chicago, IL, USA). Two-group comparisons were analyzed with Student's t-test. Comparisons among more than two groups were analyzed with one-way analysis of variance. A $\mathrm{P}<0.05$ was considered to indicate a statistically significant difference.

\section{Results}

Immunophenotyping of BMSCs. Flow cytometry was used to determine the phenotype of the BMSCs through analysis of cell-surface markers. The results revealed positive expression of CD90 and CD29 and negative expression of CD45 marker of hemopoietic stem cells. The characteristics of the cells was in accordance with a BMSC phenotype.

Effects of infrasound on BMSC proliferation. The OD of the groups receiving 10, 30, 60, 90 and $120 \mathrm{~min}$ infrasound or air treatment was measured by the CCK method (Fig. 1). The results revealed significant differences in the OD among the $10,30,60,90$ and $120 \mathrm{~min}$ infrasound groups $(\mathrm{P}<0.05)$. The maximum OD was observed in the group receiving 60 min infrasound. The mean ODs for each of the five control subgroups were observed to be sequentially reduced with increasing treatment times. When the $60 \mathrm{~min}$ infrasound and control treatments were compared, a significant elevation in the OD was detected in the infrasound group $(\mathrm{P}<0.01)$. A significant difference was also observed between the $30 \mathrm{~min}$ infrasound group and the corresponding control group $(\mathrm{P}<0.05)$; however, the $60 \mathrm{~min}$ infrasound group exhibited the greatest level of proliferation in BMSCs.

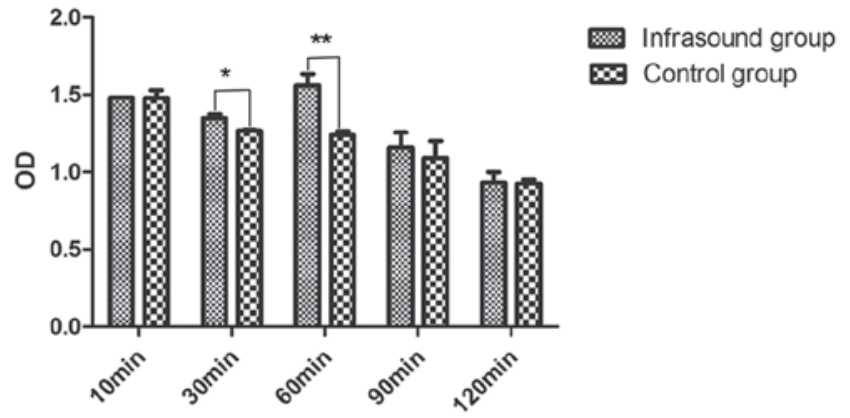

Figure 1. Proliferation of bone marrow mesenchymal stem cells (BMSCs). In order to elucidate the effects of infrasound on BMSCs, five treatment durations were used. Durations of 30 and $60 \mathrm{~min}$ infrasound induced greater BMSC proliferation as compared with those of the respective control groups. The mean optical density (OD) of the five control treatment subgroups was reduced with increasing treatment duration. No difference was detected in the 10, 90 and $120 \mathrm{~min}$ infrasound groups, or the corresponding control groups. The data indicated that $60 \mathrm{~min}$ was the most suitable duration of infrasound treatment to promote proliferation. Three independent assays were performed per group and the bars represent the means \pm standard deviation; ${ }^{*} \mathrm{P}<0.05,{ }^{* *} \mathrm{P}<0.01$.

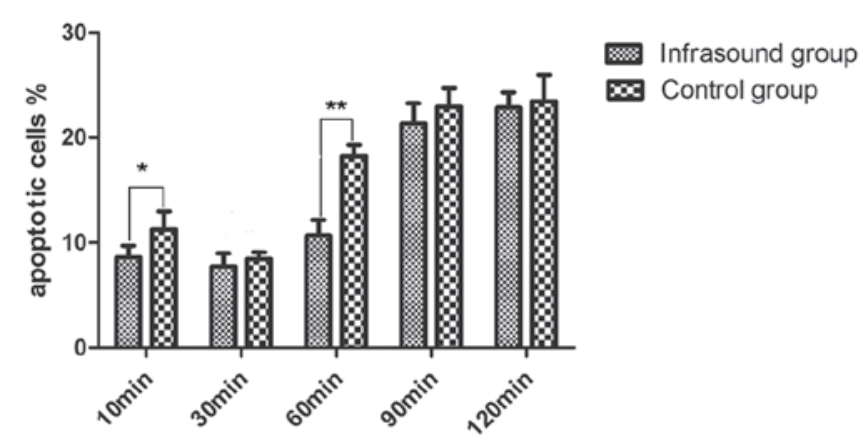

Figure 2. Apoptotic rate of bone marrow mesenchymal stem cells (BMSCs). Flow cytometry was used to analyze the effects of infrasound on BMSC apoptosis. Durations of 10 and $60 \mathrm{~min}$ infrasound treatment inhibited BMSC apoptosis. A pattern of a progressive increase in the apoptotic rate and treatment duration was observed in the control groups. No difference was identified among the 0,90 and $120 \mathrm{~min}$ infrasound groups and the corresponding control groups. The $60 \mathrm{~min}$ infrasound group exhibited significantly reduced apoptosis as compared with the 60 min control group. Three independent assays were performed per group and the bars represent the means \pm standard deviation; ${ }^{*} \mathrm{P}<0.05,{ }^{* *} \mathrm{P}<0.01$.

Effects of infrasound on BMSC apoptosis. BMSC apoptosis was analyzed by flow cytometry (Fig. 2). The apoptotic rates of BMSCs in the infrasound groups were compared with those of the respective control groups; the results revealed significant reductions in apoptosis in the 10 and $60 \mathrm{~min}$ infrasound groups 

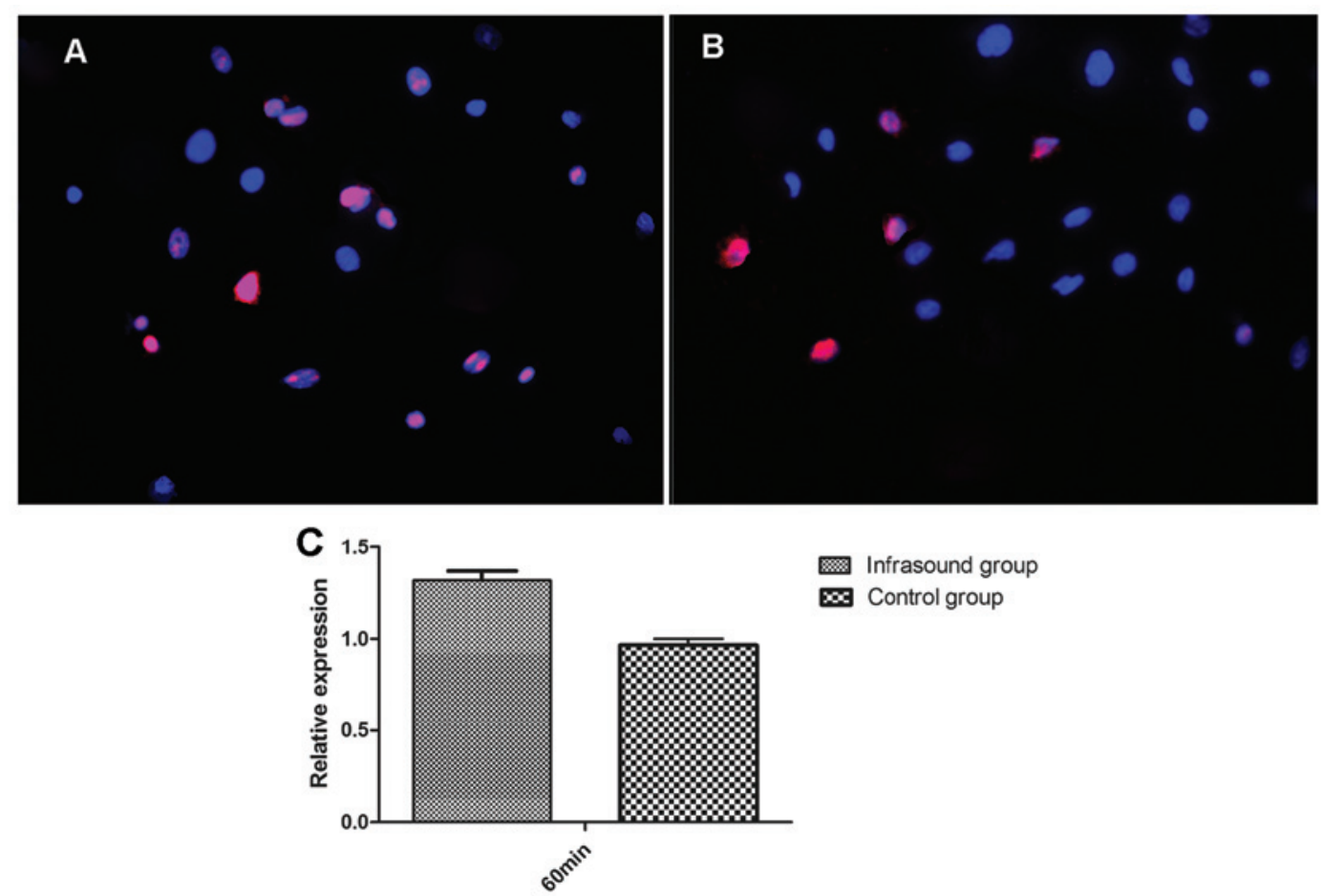

Figure 3. Expression levels of survivin protein and mRNA expression after $60 \mathrm{~min}$ infrasound or control treatment. Immunofluorescence was used to detect survivin protein expression levels in bone marrow mesenchymal stem cells (BMSCs). The percentage of positive-staining BMSCs was (A) $59.9 \pm 6.1$ in the infrasound group and (B) 24.3 \pm 5.8 in the control group $(\mathrm{P}<0.05)$. (C) Qunatitative polymerase chain reaction was used to measure survivin mRNA expression levels. The relative survivin mRNA expression levels in the infrasound group were significantly higher as compared with those in the control group $(\mathrm{P}<0.01)$.

as compared with the respective control groups. Notably, no differences in the level of apoptosis was detected in the $30 \mathrm{~min}$ infrasound group as compared with the 30 min control group $(\mathrm{P}>0.05)$. A pattern of progressive increases in the percentage of apoptotic cells with increasing treatment time was observed in the control groups. No difference was identified between the 90 and 120 min infrasound groups and the corresponding control groups. The evidence that the apoptotic rate of the cells receiving $60 \mathrm{~min}$ infrasound exhibited a significant reduction as compared with the $60 \mathrm{~min}$ control group, indicated that 60 min may be a suitable duration for infrasound treatment to inhibit BMSC apoptosis.

Effects of infrasound on the protein and $m R N A$ expression levels of survivin. As determined by the results of the proliferation and apoptosis assays of BMSCs, $60 \mathrm{~min}$ was considered the most suitable treatment duration. Therefore, only the $60 \mathrm{~min}$ infrasound and control groups were used for the measurement of survivin expression levels. The fluorescence intensity of survivin and the percentage of positive-staining BMSCs in the infrasound group were significantly higher than those of the control group ( $\mathrm{P}<0.05$; Fig. $3 \mathrm{~A}$ and $\mathrm{B})$. The relative expression level of survivin mRNA in the infrasound group was significantly increased as compared with that of the control group (Fig. 3C). These data suggest that infrasound enhances survivin expression levels.

\section{Discussion}

Mechanical stimulation through infrasound is a controversial therapeutic method. Organisms may be considered as mechan- ical vibration systems themselves and certain parts of the body exhibit natural frequencies within the range of infrasound frequency; thus infrasound may produce resonance reactions in the body, resulting in physical changes and chemical reactions (19). Due to marked penetration and low attenuation properties during long distance propagation (20-22), infrasound of BMSCs may continue to exert effects subsequent to transplantation of the cells into the body. Therefore the aim of the present study was to investigate the effects of infrasound with a low sound pressure level $(<90 \mathrm{~dB})$ on BMSCs in vitro.

BMSCs, also known as marrow mesenchymal cells or mesenchymal progenitor cells, are defined as self-renewable, multipotent progenitor cells with the capacity to differentiate into several distinct lineages (23), such as adipocytes, osteoblasts, vascular-smooth muscle-like cells and neuron-like cells (24). A number of studies have focused on transplantation of BMSCs to replace impaired tissues (25-27). Due to the changes in the microenvironments of damaged tissues, poor BMSC survival rates have been observed following BMSC migration into the tissues (28-30). Therfore, improvement in the capacity of BMSCs to survive ischemic and hypoxic conditions is required. As determined by a previous infrasound study, infrasound was hypothesized to exert protective effects on BMSCs (12). The protective effects of infrasound were, to the best of our knowledge, demonstrated for the first time in the present study.

To determine the effects of different infrasound durations on BMSCs, the cells were divided into infrasound and control groups with 10, 30, 60, 90 and 120 min treatment, and proliferation and apoptosis were measured in each group. The $10 \mathrm{~min}$ infrasound treatment was found to inhibit BMSC apoptosis but 
exerted no effect on BMSC proliferation. Conversely, 30 min infrasound promoted the proliferation of BMSCs but exerted no effect on BMSC apoptosis. Treatments for 90 and 120 min in the infrasound and control groups induced a high apoptosis and low proliferation rate. The data demonstrated that $60 \mathrm{~min}$ infrasound, which promoted the proliferation of BMSCs and inhibited apoptosis, appeared to be the most suitable treatment duration. Additionally, longer durations of air treatment induced less cell proliferation and greater apoptosis. Although the interference factor, was not identified, there was evidence of the protective effects of infrasound on BMSCs.

A previous study has indicated that the apoptosis-inducing effects of higher frequencies of infrasound on cardiac myocytes are mediated by upregulating the expression of proapoptotic proteins and downregulating the expression of anti-apoptotic proteins (31). Another study suggested that survivin expression levels are cell cycle-dependent and may inhibit cell apoptosis (16). Thus, further studies are required to investigate the survivin response to a variety of different frequencies of ultrasound.

In the present study, a suitable duration of infrasound treatment, at a frequency of 4-20 $\mathrm{Hz}$ and sound pressure level of $79-86 \mathrm{~dB}$, was found to be $60 \mathrm{~min}$. Therefore, $60 \mathrm{~min}$ may be considered as a standard reference time of BMSC infrasound treatment at this frequency. In order to elucidate the association between the protective effects of infrasound on BMSCs and survivin expression levels, the expression levels of survivin in the $60 \mathrm{~min}$ infrasound and control groups were analyzed by immunofluorescence and qPCR. Survivin is a member of the IAP family and is specifically expressed at the G2/M cell cycle phase. A number of studies have investigated the function of survivin. For example, overexpression of survivin may augment thymocyte proliferation (13). When MSCs were co-cultured with U299 and H299 myeloma cell lines, the MSCs exerted an anti-apoptotic effect on the myeloma cells, which was mediated by survivin (32). This evidence indicates that survivin promotes the proliferation of cells and inhibits apoptosis. The data from the survivin immunofluorescence assay of BMSCs in the present study revealed that the fluorescence intensity, and thus the survivin expression levels, were significantly greater in the infrasound group than in the control group. In addition, the expression levels of survivin mRNA in the infrasound group were significantly higher than those in the control group. The results demonstrated that infrasound enhances survivin expression levels. The change in survivin expression levels between the two groups may be partly responsible for the protective effect of infrasound on BMSCs. Due to the complex process of survivin expression (33) and the effects of the Notch signaling pathway on proliferation and apoptosis (34), it remains unclear whether the change in survivin expression levels induced by infrasound accounts directly for the effects of infrasound on the BMSCs. Inhibition of the signaling pathway regulating survivin expression may be performed to further analyze this association in future studies.

In conclusion, the present study confirms the protective effects of infrasound on BMSCs. Further studies are required to focus on the protective effects of infrasound on BMSC transplantation in the treatment of cerebral ischemia, as other studies have indicated that BMSCs repair the ischemic zone $(35,36)$. Evidence that hypoxia induces BMSC differentiation (37) and that infrasound enhances the viability of BMSCs indicate that infrasound may be a supplementary method in the transplantation of BMSCs to treat cerebral ischemia.

\section{Acknowledgements}

The authors would like to thank colleagues at the Department of Rehabilitation Medicine of Nanfang Hospital and Dr Yingli Bi for guidance with the experimental methods. The Experimental Center of Nanfang Hospital provided the facilities for this study.

\section{References}

1. Xiao Y, Mareddy S and Crawford, R: Clonal characterization of bone marrow derived stem cells and their application for bone regeneration. Int J Oral Sci 2: 127-135, 2006.

2. Chamberlain G, Fox J, Ashton B and Middleton J: Concise review: mesenchymal stem cells: their phenotype, differentiation capacity, immunological features, and potential for homing. Stem Cells 25: 2739-2749, 2007.

3. Bliss T, Guzman R, Daadi M and Steinberg GK: Cell transplantation therapy for stroke. Stroke 38: 817-826, 2007.

4. Parr AM, Tator $\mathrm{CH}$ and Keating A: Bone marrow-derived mesenchymal stromal cells for the repair of central nervous system injury. Bone Marrow Transplant 40: 609-619, 2007.

5. Zeng X, Yu SP, Taylor T, et al: Protective effect of apelin on cultured rat bone marrow mesenchymal stem cells against apoptosis. Stem Cell Res 8: 357-367, 2012.

6. Vaquero J, Otero L, Bonilla C, et al: Cell therapy with bone marrow stromal cells after intracerebral hemorrhage: impact of platelet-rich plasma scaffolds. Cytotherapy 15: 33-43, 2013.

7. Jagodzinski M, Breitbart A, Wehmeier M, et al: Influence of perfusion and cyclic compression on proliferation and differentiation of bone marrow stromal cells in 3-dimensional culture. J Biomech 41: 1885-1891, 2008.

8. Riddle RC, Hippe KR and Donahue HJ: Chemotransport contributes to the effect of oscillatory fluid flow on human bone marrow stromal cell proliferation. J Orthop Res 26: 918-924, 2008.

9. Leventhall G: What is infrasound? J Prog Biophys Mol 93: 130-137, 2007.

10. Pei Z, Sang H, Li R, et al: Infrasound-induced hemodynamics, ultrastructure, and molecular changes in the rat myocardium. Environ Toxicol 22: 169-175, 2007.

11. Liu ZH, Chen JZ, Ye L, et al: Effects of infrasound at $8 \mathrm{~Hz} 90 \mathrm{~dB}$ $130 \mathrm{~dB}$ on NMDAR1 expression and changes in intracellular calcium ion concentration in the hippocampus of rats. Mol Med Rep 3: 917-921, 2010.

12. Li C, Fan JZ and Wu HY: Effects of infrasound with low sound pressure level on rats with cerebral ischemia-reperfusion injury. Chinese J Rehabil Med 2009: 419-412, 2009 (In Chinese).

13. Hikita S, Hatano M, Inoue A, et al: Overexpression of TIAP/m-survivin in thymocytes enhances cell proliferation. Mol Immunol 39: 289-298, 2002.

14. Otaki M, Hatano M, Kobayashi K, et al: Cell cycle-dependent regulation of TIAP/m-survivin expression. Biochim Biophys Acta 1493: 188-194, 2000

15. Altieri DC: The case for survivin as a regulator of microtubule dynamics and cell-death decisions. Curr Opin Cell Biol 18: 609-615, 2006.

16. Yaghoobi MM and Mahani MT: NGF and BDNF expression drop off in neurally differentiated bone marrow stromal stem cells. Brain Res 1203: 26-31, 2008.

17. Charrière K, Risold PV and Fellmann D: In vitro interactions between bone marrow stromal cells and hippocampal slice cultures. C R Biol 333: 582-590, 2010.

18. Polisetti N, Chaitanya VG, Babu PP and Vemuganti GK: Isolation, characterization and differentiation potential of rat bone marrow stromal cells. Neurol India 58: 201-208, 2010.

19. Zhuang ZQ, Pei ZH and Chen JZ: The underlying mechanisms for infrasonic bioeffects. Chin J Dis control Prev 9: 328-330, 2005. 
20. Du F, Yin L, Shi M, et al: Involvement of microglial cells in infrasonic noise-induced stress via upregulated expression of corticotrophin releasing hormone type 1 receptor. Neuroscience 167: 909-919, 2010.

21. Arabadzhi VI: Infrasound and biorhythms of the human brain. Biofizika 37: 150-151, 1992 (In Russian).

22. Backteman O, Köhler J and Sjoberg L: Infrasound - tutorial and review: Part 4. J Low Freq Noise Vib 3: 96-113, 1984

23. Reis LA, Borges FT, Simões MJ, et al: Bone marrow-derived mesenchymal stem cells repaired but did not prevent gentamicin-induced acute kidney injury through paracrine effects in rats. PLoS One 7: e44092, 2012.

24. Rochefort GY, Delorme B, Lopez A, et al: Multipotential mesenchymal stem cells are mobilized into peripheral by blood hypoxia. Stem Cells 24: 2202-2208, 2006.

25. Al Fageh H, Nor Hamdam BM, Chen HC, Aminuddin BS and Ruszymah BH: The potential of intra-articular injection of chondrogenic-induced bone marrow stem cells to retard the progression of osteoarthritis in a sheep model. Exp Gerontol 47: 458-464, 2012.

26. Nishida H, Nakayama M, Tanaka H, et al: Safety of autologous bone marrow stromal cell transplantation in dogs with acute spinal cord injury. Vet Surg 41: 437-442, 2012.

27. Shin JW,Lee JK, Lee JE, et al: Combined effects of hematopoietic progenitor cell mobilization from bone marrow by granulocyte colony stimulating factor and AMD3100 and chemotaxis into the brian using stromal cell-derived factor- $1 \alpha$ in an Alzheimer's disease mouse model. Stem Cells 29: 1075-1089, 2011.

28. Resmanchi N, Floyd CL, Berman RF and Lyeth BG: Cell death and long-term maintenance of neuron-like state after differentiation of rat bone marrow stromal cells: a comparison of protocols. Brain Res 991: 46-55, 2003.
29. Li HM, Liu L, Mei X and Zhao X: Investigation on long-term survival of transplanted bone marrow mesenchymal stem cells in infarcted myocardium of rats. Chin J Cardiol 39: 171-175, 2011 (In Chinese).

30. Boukhechba F, Balaquer T, Bouvet-Gerbettaz S, et al: Fate of bone marrow stromal cells in a syngenic mode of bone formation. Tissue Eng Part A 17: 2267-2278, 2011.

31. Pei ZH, Chen BY, Tie R, et al: Infrasound exposure induces apoptosis of rat cardiac myocytes by regulating the expression of apoptosis-related proteins. Cardiovasc Toxicol 11: 341-346, 2011.

32. Wang $X$, Zhang $Z$ and Yao C: Survivin is upregulated in myeloma cell lines cocultured with mesenchymal stem cells. Leuk Res 34: 1325-1329, 2010.

33. Tang L, Ling X, Liu W, et al: Transcriptional inhibition of p21WAF1/CIP1 gene (CDKN1) expression by survivin is at least partially p53-dependent: evidence for survivin acting as a transcription factor or co-factor. Biochem Biophys Res Commun 421: 249-254, 2012.

34. Mizuguchi T, Hui T, Palm K, et al: Enhanced proliferation and differentiation of rat hepatocytes cultured with bone marrow stromal cells. J Cell Physiol 189: 106-119, 2001.

35. Shen LH, Li Y, Chen J, et al: Therapeutic benefit of bone marrow stromal cells administered 1 month after stroke. J Cereb Blood Flow Metab 27: 6-13, 2007.

36. Kawabori M, Kuroda S, Ito M, et al: Timing and cell dose determine therapeutic effects of bone marrow stromal cell transplantation in rat model of cerebral infarct. Neuropathology 33: 140-148, 2013.

37. Shin JM, Kim J, Kim HE, et al: Enhancement of differentiation efficiency of hESCs into vascular lineage cells in hypoxia via a paracrine mechanism. Stem Cell Res 7: 173-185, 2011. 